\title{
Promoter Methylation and Silencing of PTEN in Gastric Carcinoma
}

\author{
Young-Hwa Kang, Hye Seung Lee, and Woo Ho Kim \\ Cancer Research Institute (Y-HK, WHK) and Department of Pathology (HSL, WHK), Seoul National University \\ College of Medicine, and BK21 Project for Medicine, Dentistry and Pharmacy (Y-HK), Seoul, Korea
}

\begin{abstract}
SUMMARY: The PTEN/MMAC1/TEP1 gene (phosphatase and tensin homolog deleted on chromosome 10/mutated in multiple advanced cancers/TGF- $\beta$ regulated and epithelial cell enriched phosphatase 1), which regulates the signaling pathways of Akt, is a novel tumor suppressor gene implicated in multiple cancers. Because a number of tumor suppressor genes are known to be silenced by aberrant promoter methylation, we examined the methylation status of the $5^{\prime}$ CpG islands of PTEN using methylation-specific PCR. The altered expression of PTEN in 310 gastric carcinomas was analyzed by immunohistochemical staining using tissue-array and clinicopathologic profiles related to PTEN expression were characterized. Of 310 consecutive gastric carcinomas, 62 cases (20\%) showed expression loss of PTEN. Altered PTEN expression was significantly associated with tumor depth and size, lymphatic invasion, advanced stage, pTNM stage, and patient survival $(p<0.001)$. The promoter methylation frequency of PTEN was found to be present in 26 (39\%) of 66 cases examined, and 19 (73\%) of 26 gastric cancer tissues showing promoter methylation exhibited the loss of PTEN expression. Abnormalities in the expression of PTEN significantly correlated with promoter methylation $(p<0.001)$. In conclusion, silencing of the PTEN gene occurs frequently in gastric carcinoma and aberrant promoter methylation is a major mechanism of silencing of the PTEN gene. The abnormalities of the PTEN gene are associated with tumor progression, metastasis, and survival. (Lab Invest 2002, 82:285-291).
\end{abstract}

$T$ he recently identified $P T E N / M M A C 1 / T E P 1$ gene is a novel tumor suppressor gene located on chromosome 10q23.3 (Myers et al, 1997). This gene was identified by three research groups in 1997 and named PTEN (phosphatase and tensin homolog deleted on chromosome 10), MMAC1 (mutated in multiple advanced cancers), or TEP1 (TGF- $\beta$ regulated and epithelial cell enriched phosphatase 1), respectively ( $\mathrm{Li}$ and Sun, 1997; Li et al, 1997; Steck et al, 1997). PTEN/MMAC1/TEP1 (hereafter, PTEN) is a dualspecificity phosphatase with homology with the adhesion molecules tensin and auxillin. PTEN dephosphorylates and inactivates phosphatidylinositol $(3,4,5)-$ triphosphate (PI-3,4,5-P3), an intracellular second messenger, which activates serine/threonine kinase, Akt, through phosphoinositide-dependent kinase (PDK1) (Dahia, 2000). Activated Akt inactivates forkhead family transcription factor (FKHR), glycogen synthase kinase (GSK3), and the proapoptotic proteins, Bad and caspase-9 (Datta et al, 1997). The phosphatase activity of PTEN down-regulates the signaling of Akt, which suppresses apoptosis and promotes cell survival (Sun et al, 1999). PTEN induces G1 cell cycle arrest (Li and Sun, 1998). PTEN inhibits focal adhesion, spreading, and migration by dephosphorylating

Received October 18, 2001.

This study was supported by 21C Frontier Functional Human Genome Project (M101KB01000101-K020102600) from the Ministry of Science and Technology, Korea.

Address reprint requests to: Dr. Woo Ho Kim, Department of Pathology, Seoul National University College of Medicine, 28 Yongon-dong, Chongno-gu, Seoul 110-799, Korea. E-mail: woohokim@snu.ac.kr focal adhesion kinase (Tamura et al, 1998). PTEN regulates tumor-induced angiogenesis (Wen et al, 2001).

Gene silencing by genetic and epigenetic alteration of the PTEN gene was recently reported in a subset of cancers. The loss of heterozygosity and mutations of PTEN have been observed in a variety of cancers, including breast cancer, kidney cancer, bladder cancer, melanoma, glioblastoma, and lung cancer (Birck et al, 2000; Tashiro et al, 1997). Germline mutations of the PTEN genes cause Cowden's syndrome, which is characterized by multiple hamartomas and an increased risk of breast and thyroid cancer (Liaw et al, 1997). Autosomal dominant diseases, such as juvenile polyposis syndrome and Bannayan-Zonana syndrome, were found to result from germline mutation of PTEN (Marsh et al, 1997). PTEN plays a role in the pathogenesis of hematologic malignancy (Dahia et al, 1999). Altered expression of PTEN was reported in endometrial cancer, prostate cancer, and melanoma (Whang et al, 1998). Aberrant promoter methylation was associated with inactivation of the PTEN gene in endometrial cancer, prostate cancer, and melanoma (Salvesen et al, 2001; Zhou et al, 2000).

DNA promoter methylation has been recently established as an alternative mechanism of transcriptional inactivation of tumor suppressor genes (Herman, 1999). Inactivation of tumor suppressor genes has been known to contribute to the abnormal proliferation, transformation, and progression in human cancers. Aberrant promoter methylation of cancer-related key genes, such as p16, E-cadherin, TIMP-3, APC, THBS1, and $h M L H 1$, has been observed frequently in 
gastric carcinoma (Kang et al, 2001; Leung et al, 2001).

To our knowledge, the role of the PTEN gene in gastric carcinoma has not been studied to date. In this study, the methylation status of the promoter $\mathrm{CpG}$ islands and altered expression were examined to address the involvement of PTEN gene alteration in gastric carcinogenesis. The relationships between PTEN gene inactivation and clinicopathologic parameters were also analyzed.

\section{Results}

\section{Altered PTEN Expression Occurs Frequently in Gastric Carcinoma}

To address the involvement of PTEN in gastric carcinoma, the abnormalities of PTEN protein expression were examined immunohistochemically in 310 consecutive gastric carcinomas. The tissue-array slide containing 60 tissue specimens was used for immunohistochemical staining. Tumor tissues and normal tissues were simultaneously stained on the same tissue-array slide. Immunohistochemical staining exhibited cytoplasmic localization of PTEN protein in normal as well as tumor cells (Fig. 1). Expressional loss of PTEN was observed in 62 (20\%) of the 310 consecutive gastric carcinomas. This finding demonstrates that alteration in PTEN expression occurs frequently in gastric carcinoma.

\section{Clinicopathologic Profiles}

The clinicopathologic parameters were characterized and their relationship with PTEN expression was analyzed. Table 1 shows the correlation between clinicopathologic parameters and PTEN expression status. Gastric carcinomas showing expressional loss of PTEN were most common in Borrmann's type III carcinoma $(p=0.001)$. The expressional loss of PTEN was significantly associated with advanced stage compared with early stage $(p<0.001)$. Fifty-seven (92\%) of 62 cases showing the expressional loss of PTEN were in the advanced stage. The altered expression of PTEN was significantly associated with tumor depth and tumor size $(p<0.01)$, lymph node metastasis, lymphatic invasion $(p<0.001)$, and pTNM stage $(p<0.001)$. However, no correlation was found between PTEN expression phenotype and distant-organ metastasis or vascular invasion. This indicates that the altered expression of PTEN is involved in the progression and lymph node metastasis of gastric carcinoma.

The immunohistochemical staining results of $\mathrm{p53}$, MUC1, MUC2, and Bcl2 proteins were analyzed to address the relationships between PTEN and the expressions of the other proteins (Table 2). The loss of PTEN expression was significantly associated with p53 overexpression $(p=0.04)$ and positive $\mathrm{Bcl} 2$ expression $(p=0.04)$. Mucin phenotypes of gastric carcinoma were also significantly correlated with PTEN expression status.

\section{Survival Analysis}

The survival of patients showing expressional loss of PTEN was remarkably worse than that of patients showing positive PTEN expression ( $p=0.001$ ) (Fig. 2). When survival curves were stratified according to disease progression using the Kaplan-Meier method, PTEN expression phenotype tended to correlate with patient survival in a subgroup of advanced carcinoma, but without significance $(p=0.08)$. Of the other clinicopathologic parameters, tumor location, tumor size, lymphatic invasion, and pTNM stage were found to correlate significantly with patient survival $(p<$ 0.01). By multivariate Cox regression model, pTNM stage was found to be significantly and independently associated with patient survival $(p<0.001)$, but PTEN expression status $(p=0.36)$ and Lauren's classification $(p=0.12)$ were not.
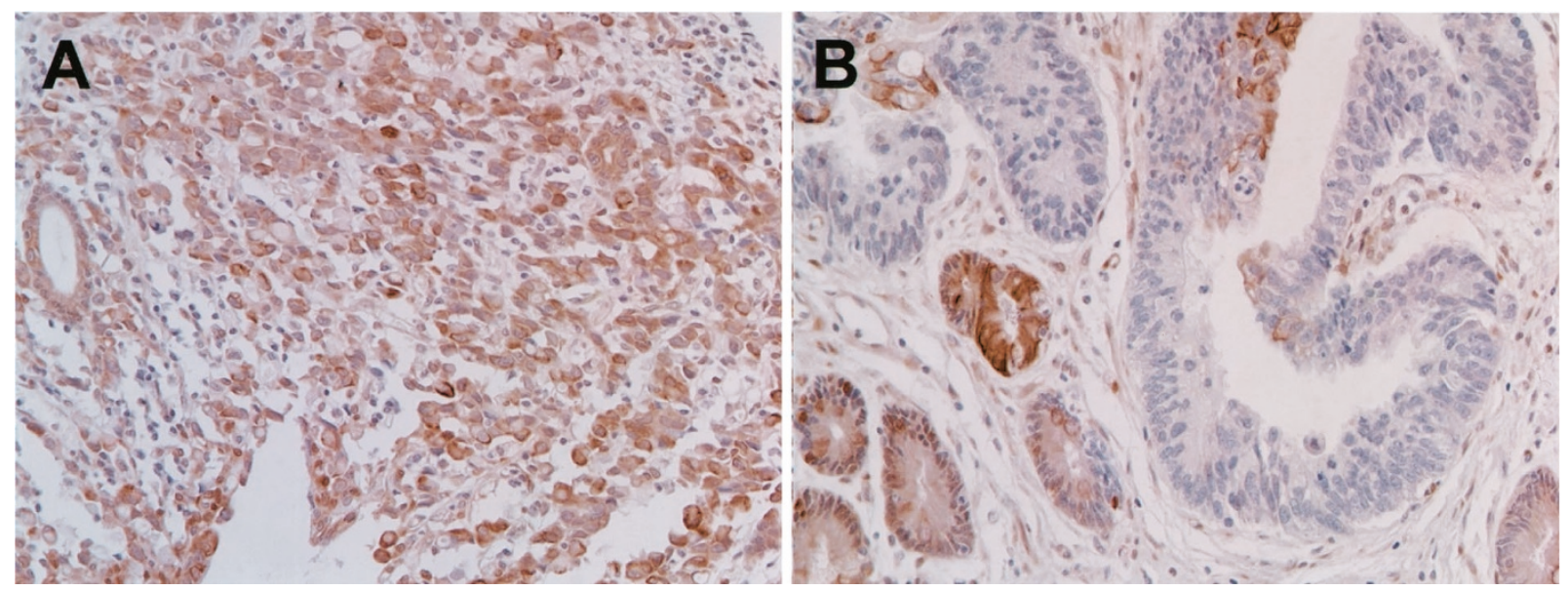

Figure 1.

Immunohistochemical staining of PTEN protein. A, Diffuse positive signals on cytoplasm of tumor cells are shown. B, PTEN protein was absent in the tumor cells, whereas adjacent noncancerous glands showed positive staining. Original magnification, $\times 200$. 
Table 1. Clinicopathologic Characteristics of PTEN Expression in Gastric Cancers

\begin{tabular}{|c|c|c|c|c|}
\hline Characteristics & $\begin{array}{c}\text { Total } \\
(n=310)\end{array}$ & $\begin{array}{l}\text { PTEN negative } \\
(n=62)\end{array}$ & $\begin{array}{l}\text { PTEN positive } \\
(n=248)\end{array}$ & $p$ value $^{a}$ \\
\hline Sex & & & & N.S. \\
\hline Male & 212 & 47 & 165 & \\
\hline Female & 98 & 15 & 83 & \\
\hline Mean age (yr) & $54.4 \pm 13.1$ & $56.8 \pm 13.4$ & $53.7 \pm 12.9$ & N.S. \\
\hline Location & & & & N.S. \\
\hline Antrum & 166 & 30 & 136 & \\
\hline Body \& Cardia & 144 & 32 & 112 & \\
\hline Tumor size $(\mathrm{cm})$ & $5.2 \pm 3.7$ & $6.5 \pm 3.4$ & $4.8 \pm 2.9$ & 0.003 \\
\hline WHO classification & & & & N.S. \\
\hline W/D and M/D & 115 & 24 & 91 & \\
\hline P/D & 131 & 30 & 101 & \\
\hline Mucinous & 17 & 0 & 17 & \\
\hline Signet ring cell & 47 & 8 & 39 & \\
\hline Lauren's classification & & & & N.S. \\
\hline Intestinal & 119 & 23 & 96 & \\
\hline Diffuse & 161 & 36 & 125 & \\
\hline Mixed & 30 & 3 & 27 & \\
\hline Lymphatic invasion & & & & $<0.001$ \\
\hline Absent & 223 & 31 & 192 & \\
\hline Present & 87 & 31 & 56 & \\
\hline Depth of invasion & & & & $<0.001$ \\
\hline EGC & 98 & 5 & 93 & \\
\hline AGC & 212 & 57 & 155 & \\
\hline Lymph node metastasis & & & & $<0.001$ \\
\hline Absent & 120 & 11 & 109 & \\
\hline Present & 190 & 51 & 139 & \\
\hline Distant-organ metastasis & & & & N.S. \\
\hline Absent & 294 & 56 & 238 & \\
\hline Present & 16 & 6 & 10 & \\
\hline PTNM stage ${ }^{b}$ & & & & $<0.001$ \\
\hline I & 138 & 13 & 125 & \\
\hline II & 60 & 10 & 50 & \\
\hline III & 68 & 23 & 45 & \\
\hline IV & 44 & 16 & 28 & \\
\hline
\end{tabular}

N.S., not significant.

${ }^{a}$ The $\chi^{2}$ test (unpaired $t$-test) or Fisher's exact test were used to compare all variables.

${ }^{b}$ Pathological stage was classified according to the criteria of the American Joint Committee on Cancer.

Table 2. Correlation Between Expression of PTEN and Expression of Other Proteins

\begin{tabular}{lccr}
\hline & \multicolumn{2}{c}{ PTEN expression } & \\
\cline { 2 - 3 } & Negative & Positive & $p$ value \\
\hline p53 overexpression & $27 / 59(46 \%)$ & $75 / 237(32 \%)$ & 0.04 \\
Bc12 expression & $2 / 58(3 \%)$ & $34 / 241(14 \%)$ & 0.04 \\
MUC1 expression & $21 / 60(35 \%)$ & $51 / 241(21 \%)$ & 0.03 \\
MUC2 expression & $7 / 59(12 \%)$ & $73 / 233(31 \%)$ & $<0.01$ \\
\hline
\end{tabular}

\section{Promoter Methylation of PTEN is a Major Alternative Mechanism of Gene Silencing}

The status of promoter methylation of PTEN in 66 gastric cancer tissues was determined using methylation-specific PCR (MSP). Aberrant promoter methylation of PTEN was detected in 26 (39\%) of 66 gastric carcinomas. Seven (27\%) of 26 primary gastric cancers with promoter methylation expressed PTEN protein, whereas 33 (83\%) of 40 gastric cancers without methylation expressed PTEN protein. Promoter methylation of PTEN was significantly correlated with the loss of PTEN protein expression $(p<$ 0.001 , Fisher's exact test), but this correlation was not completely consistent (Table 3 ). These findings suggest that the silencing of the PTEN gene can be caused by promoter methylation as well as by other inactivating mechanisms. The unmethylated form was consistently found in tumor tissue because of normal cell contamination (Fig. 3).

\section{Discussion}

The role of PTEN identified as a novel tumor suppressor gene has been studied in a variety of cancers. Loss of heterozygosity and mutation of PTEN have been reported in melanoma, glioblastoma, kidney 
A

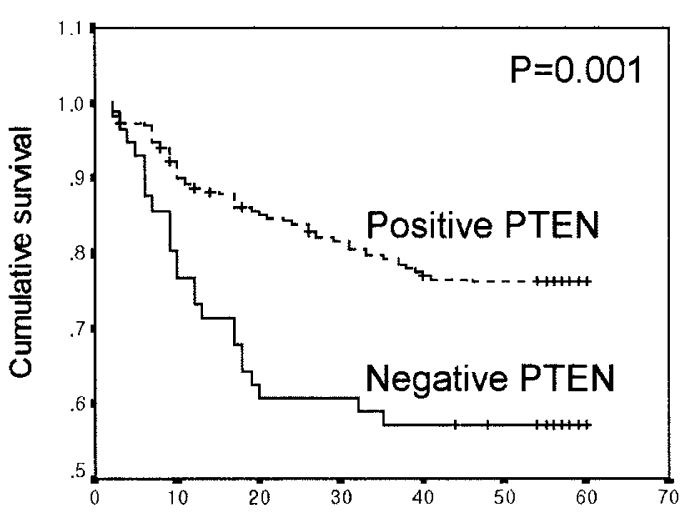

Follow-up months
B

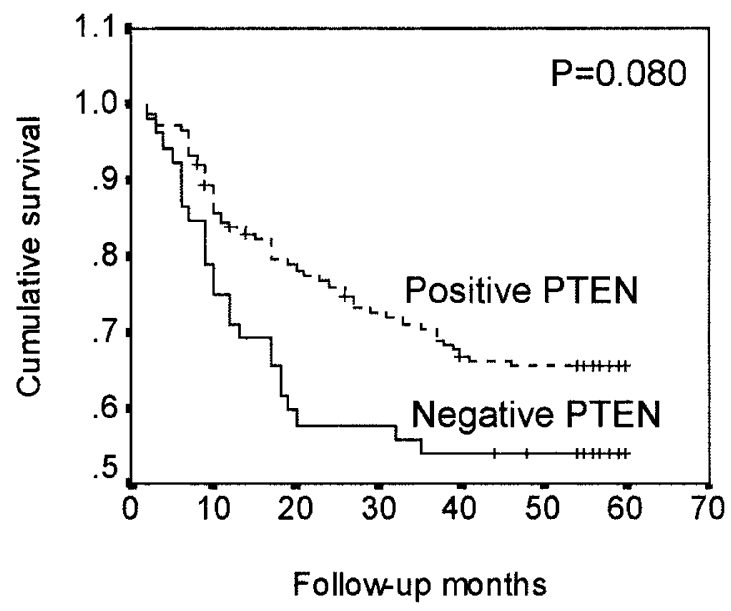

Figure 2.

The effects of PTEN expression on patient survival. A, Survival curves by the Kaplan-Meier method. PTEN-negative tumors have an unfavorable prognosis as compared with PTEN-positive tumors $(p=0.001)$. B, Survival curves of advanced stage cancers by the Kaplan-Meier method. PTEN expression is correlated with patient survival in advanced cancers, but without significance $(p=0.080)$. $p$ was determined using the log-rank test.

cancer, lung cancer, and breast cancer (Rhei et al, 1997; Risinger et al, 1998; Teng et al, 1997). In particular, the mutation of PTEN appears with a frequency of up to $50 \%$ in endometrial cancer (Tashiro et al, 1997) compared with other cancers, including glioblastoma (28\%), prostate cancer (30\%), and melanoma (15\%) (Dahia, 2000). Abnormalities in the expression of the PTEN gene have been shown in esophageal cancer, melanoma, and prostate cancer (Cairns et al, 1997; Whang et al, 1998). Gene silencing by promoter methylation of PTEN has been reported in endometrial and prostate cancer (Salvesen et al, 2001). Aberrant promoter methylation has been suggested as a potential mechanism of PTEN inactivation.

DNA methylation plays an important role in the gene regulation of mammals (Razin and Riggs, 1980) and transcriptional inactivation is a recognized major mechanism for the loss of function in the tumor suppressor gene. Promoter methylation is a representative example of the transcriptional silencing of tumor suppressor genes (Baylin et al, 1998; Herman, 1999). Cancer-related genes, such as APC, p16, p15, E-cadherin, hMLH1, and TIMP3, have been inactivated by aberrant promoter methylation in gastric carcinoma (Leung et al, 2001; Tsuchiya et al, 2000). The role of PTEN in gastric carcinogenesis has not been reported to date. To address the involvement of alterations of the PTEN gene in gastric carcinoma, we

Table 3. Correlation Between PTEN Expression and Promoter Methylation

\begin{tabular}{lcc}
\hline & \multicolumn{2}{c}{ Methylation-specific PCR } \\
\cline { 2 - 3 } PTEN expression & Unmethylated & Methylated \\
\hline Positive & 33 & 7 \\
Negative & 7 & 19 \\
\hline$p<0.001$. &
\end{tabular}

examined the promoter methylation and the altered expression of the PTEN gene. Aberrant promoter methylation of PTEN was detected in 26 (39\%) of 66 primary gastric cancers. Promoter methylation of PTEN was significantly correlated with the expressional loss of the PTEN protein $(p<0.001$, Fisher's exact test). Nineteen (73\%) of 26 gastric cancer tissues showing promoter methylation in MSP revealed the loss of PTEN expression, whereas 7 (18\%) of 40 gastric cancers showing no methylation exhibited the expressional loss of PTEN. This may indicate that the gene inactivation of PTEN is caused by promoter methylation as well as by other inactivating mechanisms, such as mutation or loss of heterozygosity.

Since the PTEN gene was recently identified as a tumor suppressor gene, study of the biological and biochemical roles of the PTEN gene has intensified. It has been reported that PTEN controls cellular processes, such as cell cycling, apoptosis, and cell death, as a regulator of phosphatidylinositol 3-kinase (PI3K) (Dahia, 2000). Although many studies on PTEN have already been reported, the role of the PTEN gene in cancer is unclear and many controversial results have been presented. Several articles on endometrial car-

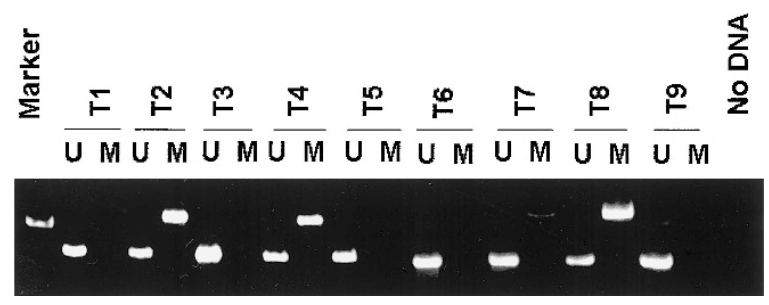

Figure 3.

Methylation-specific PCR of PTEN in gastric cancer tissue. The unmethylated (162 bp) and methylated (206 bp) PCR products were designated as $U$ or $M$, respectively. T2, T4, and T8 exhibited PCR products by methylated primers. All tumor samples showed the unmethylated form because of normal cell contamination. 
cinoma have shown that altered PTEN expression is associated with an early stage in endometrial cancer (Mutter et al, 2000) and that inactivation of PTEN is associated with nonmetastatic disease and favorable clinical characteristics (Risinger et al, 1998). In contrast, other papers have shown that gene silencing of PTEN is associated with advanced stage and metastasis in endometrial and prostate carcinoma (Rasheed et al, 1997; Whang et al, 1998). It was reported that mutation of the PTEN gene correlated with tumor progression and poor outcome in cervical cancer, breast cancer, and melanoma (Depowski et al, 2001; Poetsch et al, 2001).

In this study, clinicopathologic parameters related to PTEN expression were characterized. The expressional loss of PTEN increased remarkably according to disease stage (pTNM Stage I, 9\%; Stage II, 16\%; Stage III, 34\%; Stage IV, 36\%). Lymph node metastasis was observed in $82 \%$ of PTEN-negative tumors compared with $52 \%$ of PTEN-positive tumors. Our data show that PTEN gene alteration is associated with advanced stage and lymph node metastasis. Our results suggest that PTEN may play an important role in the regulation of tumor progression and metastasis during the development of gastric carcinoma. Gastric carcinoma patients showing expressional loss of PTEN showed poor prognosis. This result suggests that PTEN is a potential prognostic factor in gastric carcinoma.

Our previous study showed that MUC1, MUC2, and p53 were prognostic markers in gastric carcinoma (Lee et al, 2001). In the present study, the loss of PTEN expression was significantly associated with p53 overexpression and a similar association was reported in glioblastoma (Kraus et al, 2000). A recent study demonstrates that loss of PTEN leads to up-regulation of the $\mathrm{Bcl} 2$ gene, thus contributing to survival of cancer cells in prostate cancer (Huang et al, 2001). Our results also show the strong association of altered PTEN expression with $\mathrm{Bcl} 2$-positive expression $(p=0.04)$ in gastric carcinoma.

In conclusion, our findings demonstrate that aberrant promoter methylation and the altered expression of the PTEN gene occur frequently in gastric carcinoma. Promoter methylation of $\mathrm{CpG}$ islands of PTEN presents a major alternative mechanism of gene silencing. Moreover, the expressional loss of PTEN correlates significantly with tumor progression, lymph node metastasis, and poor survival. Our results suggest that PTEN probably plays an important role in the progression of gastric carcinoma and that the expressional phenotype of PTEN may be a potential prognostic factor in gastric carcinoma.

\section{Materials and Methods}

\section{Patients and Samples}

Three hundred and ten specimens of gastric cancer tissues, resected surgically at the Seoul National University College of Medicine, were used for this study. The age, sex, tumor location, tumor size, lymphatic invasion, and PTNM stage were evaluated by reviewing medical charts and pathologic records. The mean age of the patients was 54.4 years. No patient had received preoperative chemo- or radiotherapy. Glass slides were reviewed for histologic classification (according to the World Health Organization's and Lauren's classification). The clinical outcome of the patients was followed from the date of surgery (1995) to the date of death or to December 1, 1999. The follow-up period was 1 month to approximately 60 months (mean, 42 months). Cases lost to follow-up and deaths from causes other than gastric cancer were regarded as censored data (3.8\%) for the analysis of survival rates. The DNA of 66 cancerous tissues selected randomly from 310 consecutive gastric carcinomas was obtained from formalin-fixed, paraffinembedded surgical blocks. DNA was extracted by the proteinase $\mathrm{K}$ digestion and phenol/chloroform/isoamyl alcohol (25:24:1) procedure.

\section{Immunohistochemical Staining}

The protein expression of PTEN was assessed by an immunohistochemical staining method using a tissue array (Superbiochips Laboratories, Seoul, Korea). Core tissue biopsies ( $2 \mathrm{~mm}$ in diameter) were taken from individual paraffin-embedded gastric tumors and seeded in a new recipient paraffin block (tissue-array block). Each tissue-array block consisted of 60 samples. In total, 6 tissue-array slides of 310 cases were used in the immunohistochemical study. Each tissuearray block contained normal gastric mucosa from body, antrum, and intestinal metaplasia as an internal control. Sections $4 \mu \mathrm{m}$ thick were cut from each tissue-array block. These were then deparaffinized and dehydrated. Immunohistochemical staining against PTEN (mouse monoclonal antibody; AG Science, San Diego, California) was performed using a streptavidin peroxidase procedure. Anti-PTEN antiserum was diluted to 1:200 with PBS. Antigen-bound primary antibody was detected using a standard avidin-biotin immunoperoxidase complex (ABC) method (Vectastain Elite ABC peroxidase kit; Vector Laboratories, Burlingame, California). Those cases with less than $10 \%$ of cytoplasmic staining in tumor cells were considered to have loss of PTEN expression. Immunohistochemical staining against p53 (mouse monoclonal antibody; DAKO, Glostrup, Denmark), Bcl2 (mouse monoclonal antibody; DAKO), MUC1 (mouse monoclonal antibody NCL-MUC-1: Novocastra Laboratories, Newcastle, United Kingdom), and MUC2 (mouse monoclonal antibody NCL-MUC-2; Novocastra) was performed and the relationships between the expression of PTEN and the other proteins were examined.

\section{Bisulfite Modification and MSP}

Genomic DNA was modified with sodium bisulfite as described previously (Herman et al, 1996). Briefly, 1 ug of genomic DNA was denatured with $0.2 \mathrm{M} \mathrm{NaOH}$ for 10 minutes at $37^{\circ} \mathrm{C}$. Then $10 \mathrm{~mm}$ of hydroquinone and 
$3 \mathrm{~m}$ of sodium bisulfite ( $\mathrm{pH}$ 5.0) were added and mixed. Samples were incubated at $50^{\circ} \mathrm{C}$ for 16 hours. DNA was purified using the Wizard DNA purification kit (Promega, Madison, Wisconsin). This procedure resulted in the conversion of unmethylated cytosine to thymine, but methylated cytosine remained unchanged. Then 1 ul of bisulfite-modified DNA was amplified using primers that specifically amplify methylated or unmethylated DNA (gene bank accession number AF067844). The primers used were as described previously (Salvesen et al, 2001). Primer sequences of PTEN were as follows: unmethylated reaction, sense primer, 5'-GTGTTGGTGGAGGTAGTTGTTT-3', antisense primer, 5'-ACCACTTAACTCTAAACCACAACCA-3'; methylated reaction, sense primer, 5'- TTCGTTCGTCGTCGTCGTATTT-3', antisense primer, 5'-GCCGCTTAACTCTAAACCGCAACCG-3'. PCR was performed as follows: one cycle of $95^{\circ} \mathrm{C}$ for 5 minutes and 35 cycles of $94^{\circ} \mathrm{C}$ for 45 seconds, $61^{\circ} \mathrm{C}$ for 30 seconds, and $72^{\circ} \mathrm{C}$ for 30 seconds, and then one cycle of $72^{\circ} \mathrm{C}$ for 10 minutes. PCR products were analyzed using nondenaturing $6 \%$ polyacrylamide gel electrophoresis and ethidium bromide staining. Normal lymphocytes were used as a negative control for unmethylated form in MSP.

\section{Statistical Analyses}

The $\chi^{2}$ test or Fisher's exact test (two sided) was used to determine the correlation between PTEN expression phenotype and the clinicopathologic parameters. Survival curves were estimated using the KaplanMeier product-limit method, and differences between the survival curves were tested using the log-rank test. A multivariate survival analysis was conducted according to the Cox proportional hazards model. Results were considered to be statistically significant when $P$ values were less than 0.05 . All statistical analyses were conducted using the SPSS 9.0 statistical software program (SPSS, Chicago, Illinois).

\section{Acknowledgements}

The authors thank Ms. S. I. Choi and Ms. S. P. Kim for technical assistance.

\section{References}

Baylin SB, Herman JG, Graff JR, Vertino PM, and Issa JP (1998). Alterations in DNA methylation: A fundamental aspect of neoplasia. Adv Cancer Res 72:141-196.

Birck A, Ahrenkiel V, Zeuthen J, Hou-Jensen K, and Guldberg P (2000). Mutation and allelic loss of the PTEN/MMAC1 gene in primary and metastatic melanoma biopsies. J Invest Dermatol 114:277-280.

Cairns P, Okami K, Halachmi S, Halachmi N, Esteller M, Herman JG, Isaacs WB, Bova GS, and Sidransky D (1997). Frequent inactivation of PTEN/MMAC1 in primary prostate cancer. Cancer Res 57:4997-5000.

Dahia PL, Aguiar RC, Alberta J, Kum JB, Caron S, Sill H, Marsh DJ, Ritz J, Freedman A, Stiles C, and Eng C (1999). PTEN is inversely correlated with the cell survival factor
Akt/PKB and is inactivated via multiple mechanisms in haematological malignancies. Hum Mol Genet 8:185-193.

Dahia PLM (2000). PTEN, a unique tumor suppressor gene. Endocr Relat Cancer 7:115-129.

Datta SR, Dudek H, Tao X, Masters S, Fu H, Gotoh Y, and Greenberg ME (1997). Akt phosphorylation of BAD couples survival signals to the cell-intrinsic death machinery. Cell 91:231-241.

Depowski PL, Rosenthal SI, and Ross JS (2001). Loss of expression of the PTEN gene protein product is associated with poor outcome in breast cancer. Mod Pathol 14:672676.

Herman JG (1999). Hypermethylation of tumor suppressor genes in cancer. Semin Cancer Biol 9:359-367.

Herman JG, Graffm JR, Myohanen S, Nelkin BD, and Baylin SB (1996). Methylation-specific PCR: A novel PCR assay for methylation status of $\mathrm{CpG}$ islands. Proc Natl Acad Sci USA 93:9821-9826.

Huang H, Cheville JC, Pan Y, Roche PC, Schmidt LJ, and Tindall DJ (2001). PTEN induces chemosensitivity in PTENmutated prostate cancer cells by suppression of $\mathrm{Bcl}-2$ expression. J Biol Chem 276: 38830-38836.

Kang GH, Shim YH, Jung HY, Kim WH, Ro JY, and Rhyu MG (2001). CpG island methylation in premalignant stages of gastric carcinoma. Cancer Res 61:2847-2851.

Kraus JA, Glesmann N, Beck M, Krex D, Klockgether T, Schackert G, and Schlegel U (2000). Molecular analysis of the PTEN, TP53 and CDKN2A tumor suppressor genes in long-term survivors of glioblastoma multiforme. J Neurooncol 48:89-94.

Lee HS, Lee HK, Kim HS, Yang HK, Kim Yl, and Kim WH (2001). MUC1, MUC2 and p53 expression in gastric carcinoma: Their role as prognostic markers. Cancer 92: 1427-1434.

Leung WK, Yu J, Ng EK, To KF, Ma PK, Lee TL, Go MY, Chung SC, and Sung JJ (2001). Concurrent hypermethylation of multiple tumor-related genes in gastric carcinoma and adjacent normal tissues. Cancer 91:2294-2301.

Li DM and Sun H (1998). PTEN/MMAC1/TEP1 suppresses the tumorigenicity and induces $\mathrm{G} 1$ cell cycle arrest in human glioblastoma cells. Proc Natl Acad Sci U S A 95:1540615411.

Li DM and Sun H (1997). TEP1, encoded by a candidate tumor suppressor locus, is a novel protein tyrosine phosphatase regulated by transforming growth factor beta. Cancer Res 57:2124-2129.

Li J, Yen C, Liaw D, Podsypanina K, Bose S, Wang SI, Puc J, Miliaresis C, Rodgers L, McCombie R, Bigner SH, Giovanella BC, Ittmann M, Tycko B, Hibshoosh $\mathrm{H}$, Wigler $\mathrm{MH}$, and Parsons R (1997). PTEN, a putative protein tyrosine phosphatase gene mutated in human brain, breast, and prostate cancer. Science 275:1943-1947.

Liaw D, Marsh DJ, Li J, Dahia PL, Wang SI, Zheng Z, Bose S, Call KM, Tsou HC, Peacocke M, Eng C, and Parsons R (1997). Germline mutations of the PTEN gene in Cowden disease, an inherited breast and thyroid cancer syndrome. Nat Genet 16:64-67.

Marsh DJ, Dahia PL, Zheng Z, Liaw D, Parsons R, Gorlin RJ, and Eng C (1997). Germline mutations in PTEN are present in Bannayan-Zonana syndrome. Nat Genet 16:333-334. 
Mutter GL, Lin MC, Fitzgerald JT, Kum JB, Baak JP, Lees JA, Weng LP, and Eng C (2000). Altered PTEN expression as a diagnostic marker for the earliest endometrial precancers. J Natl Cancer Inst 92:924-930.

Myers MP, Stolarov J, Eng C, Li J, Wang SI, Wigler MH, Parsons R, and Tonks NK (1997). PTEN, the tumor suppressor from human chromosome 10q23, is a dual specificity phosphatase. Proc Natl Acad Sci USA 94:9052-9057.

Poetsch M, Dittberner T, and Woenckhaus C (2001). PTEN/ MMAC1 in malignant melanoma and its importance for tumor progression. Cancer Genet Cytogenet 125:21-26.

Rasheed BK, Stenzel TT, McLendon RE, Parsons R, Friedman AH, Friedman HS, Bigner DD, and Bigner SH (1997). PTEN gene mutations are seen in high-grade but not in low-grade gliomas. Cancer Res 57:4187-4190.

Razin A and Riggs AD (1980). DNA methylation and gene regulation. Science 210:604-610.

Rhei E, Kang L, Bogomolniy F, Federici MG, Borgen PI, and Boyd $J$ (1997). Mutation analysis of the putative tumor suppressor gene PTEN/MMAC1 in primary breast carcinomas. Cancer Res 57:3657-3659.

Risinger JI, Hayes K, Maxwell GL, Carney ME, Dodge RK, Barrett JC, and Berchuck A (1998). PTEN mutation in endometrial cancers is associated with favorable clinical and pathologic characteristics. Clin Cancer Res 4:3005-3010.

Salvesen HB, MacDonald N, Ryan A, Jacobs IJ, Lynch ED, Akslen LA, and Das S (2001). PTEN methylation is associated with advanced stage and microsatellite instability in endometrial carcinoma. Int J Cancer 91:22-26.

Steck PA, Pershouse MA, Jasser SA, Yung WK, Lin H, Ligon AH, Langford LA, Baumgard ML, Hattier T, Davis T, Frye C, Hu R, Swedlund B, Teng DH, and Tavtigian SV (1997). Identification of a candidate tumour suppressor gene, MMAC1, at chromosome 10q23.3 that is mutated in multiple advanced cancers. Nat Genet 15:356-362.

Sun H, Lesche R, Li DM, Liliental J, Zhang H, Gao J, Gavrilova N, Mueller B, Liu X, and Wu H (1999). PTEN modulates cell cycle progression and cell survival by regulating phosphatydylinositol 3,4,5,-tirphosphate and AKT/ protein kinase B signaling pathway. Proc Natl Acad Sci USA 96:6199-6204.
Tamura M, Gu J, Matsumoto K, Aota S, Parsons R, and Yamada KM (1998). Inhibition of cell migration, spreading, and focal adhesions by tumor suppressor PTEN. Science 280:1614-1617.

Tashiro H, Blazes MS, Wu R, Cho KR, Bose S, Wang SI, Li J, Parsons R, and Ellenson LH (1997). Mutations in PTEN are frequent in endometrial carcinoma but rare in other common gynecological malignancies. Cancer Res 57:3935-3940.

Teng DH, Hu R, Lin H, Davis T, lliev D, Frye C, Swedlund B, Hansen KL, Vinson VL, Gumpper KL, Ellis L, El-Naggar A, Frazier M, Jasser S, Langford LA, Lee J, Mills GB, Pershouse MA, Pollack RE, Tornos C, Troncoso P, Yung WK, Fujii G, Berson A, and Steck PA (1997). MMAC1/PTEN mutations in primary tumor specimens and tumor cell lines. Cancer Res 57:5221-5225.

Tsuchiya T, Tamura G, Sato K, Endoh Y, Sakata K, Jin Z, Motoyama T, Usuba O, Kimura W, Nishizuka S, Wilson KT, James SP, Yin J, Fleisher AS, Zou T, Silverberg SG, Kong D, and Meltzer SJ (2000). Distinct methylation patterns of two APC gene promoters in normal and cancerous gastric epithelia. Oncogene 19:3642-3646.

Wen S, Stolarov J, Myers MP, Su JD, Wigler MH, Tonks NK, and Durden DL (2001). PTEN controls tumor-induced angiogenesis. Proc Natl Acad Sci USA 98:4622-4627.

Whang YE, Wu X, Suzuki H, Reiter RE, Tran C, Vessella RL, Said JW, Isaacs WB, and Sawyers CL (1998). Inactivation of the tumor suppressor PTEN/MMAC1 in advanced human prostate cancer through loss of expression. Proc Natl Acad Sci USA 95:5246-5250.

Zhou XP, Gimm O, Hampel H, Niemann T, Walker MJ, and Eng C (2000). Epigenetic PTEN silencing in malignant melanomas without PTEN mutation. Am J Pathol 157:1123-1128. 